\title{
Healthrelated quality of life in patients with psoriatic and rheumatoid arthritis: data from the prospective multicenter NOR-DMARD study compared with Norwegian general population controls
}

Brigitte Michelsen ${ }^{1,2}$, Till Uhlig ${ }^{1,3}$, Joseph Sexton ${ }^{1}$, Désirée van der Heijde ${ }^{1,4}$, Hilde Berner Hammer $^{1}$, Eirik Klami Kristianslund ${ }^{1}$, Ada Wierød ${ }^{5}$, Gunnstein Bakland ${ }^{6}$, Erik Rødevand ${ }^{7}$, Frode $\mathrm{Kr}_{\varnothing} \mathrm{II}^{8}$, Jon Håvard Loge ${ }^{3}$, Glenn Haugeberg ${ }^{9,10}$, Tore K Kvien ${ }^{1,3}$

${ }^{1}$ Department of Rheumatology, Diakonhjemmet Hospital, Oslo, Norway; 2 Unit of Rheumatology, Department of Medicine, Hospital of Southern Norway Trust, Kristiansand, Norway; ${ }^{3}$ Faculty of Medicine, University of Oslo, Oslo, Norway; ${ }^{4}$ Department of Rheumatology, Leiden University Medical Center, Leiden, The Netherlands; ${ }^{5}$ Department of Rheumatology, Vestre Viken/Drammen Hospital, Drammen, Norway; ${ }^{6}$ Department of Rheumatology, University Hospital of Northern Norway, Troms $\varnothing$, Norway; ${ }^{7}$ Department of Rheumatology, St. Olavs Hospital, Trondheim, Norway; ${ }^{8}$ Department of Rheumatology, Lillehammer Hospital for Rheumatic Diseases, Lillehammer, Norway; ${ }^{9}$ Department of Rheumatology, Martina Hansens Hospital, Bærum, Norway; ${ }^{10}$ Norwegian University of Science and Technology, Trondheim, Norway

Correspondence to: Brigitte Michelsen, Department of Rheumatology, Diakonhjemmet Hospital, Box 23 Vinderen, Oslo, Norway; brigitte_michelsen@yahoo.no

Key words: Rheumatoid arthritis, psoriatic arthritis, outcomes research, patient perspective

Word count: 1595 


\section{ABSTRACT}

\section{Objectives}

To compare 1) Short Form-36 (SF-36) Physical (PCS) and Mental (MCS) Component

Summaries, scale scores and Short Form- 6 dimensions (SF-6D) between rheumatoid (RA) and psoriatic arthritis (PsA) patients and Norwegian general population controls and 2)

improvements in these measures between RA and PsA patients.

\section{Methods}

ANCOVA analyses were performed to compare SF-36 measures between first-time enrolled RA $(n=3898)$ and PsA ( $n=1515)$ patients from the prospective observational multicenter NORDMARD study (6 months follow-up) and general population controls ( $n=2323$ ).

\section{Results}

In age and gender-adjusted analyses PsA vs. RA patients had similar PCS, MCS and SF-6D $(p \geq 0.14)$, worse vitality and general health, but better physical functioning at $0 / 6$ months $(p \leq 0.03)$. With additional DAS28-adjustment as a proxy for joint inflammation, PCS, most scale scores and SF-6D were worse in PsA vs. RA patients at $0 / 3 / 6$ months $(p \leq 0.01)$. PCS was more impaired than MCS both in RA and PSA compared with general population controls $(p \leq 0.001)$. Mean 3- and 6-month improvements after DMARD treatment were larger in RA vs. PsA patients for bodily pain, vitality and mental health ( $p \leq 0.02)$.

\section{Conclusions}

HRQoL was overall similar in RA and PsA patients - with a tendency to worse scores in PsA and worse compared with general population controls.

\section{INTRODUCTION}

Health-Related Quality of Life (HRQoL) is part of the core set of data to be collected in patients with psoriatic arthritis (PSA) and recognized to be of major importance also in other rheumatic diseases, including rheumatoid arthritis (RA) ${ }^{1,2} \mathrm{HRQ}$ oL has been found to be impaired in patients with inflammatory arthritides compared with the general population. ${ }^{3-6}$ 
In smaller observational studies performed 1-2 decades ago similar HRQoL in RA and PsA patients were described, ${ }^{7,8}$ but also differences in Short Form-36 (SF-36) scale scores. ${ }^{5}$ To our knowledge no large, prospective observational study has compared HRQoL between RA and PsA patients and the general population using the widely recognized SF-36.

The aim of this study was to compare SF-36 Physical and Mental Component Summaries (PCS, MCS), scale scores as well as short form-6 dimensions utility score (SF-6D) between RA and PsA patients from a large prospective observational study, as well as with general population controls. Furthermore, we aimed to compare improvements in PCS, MCS and scale scores between RA and PsA patients from baseline to 3 and 6 months follow-up after initiation of treatment with disease-modifying anti-rheumatic drugs (DMARDs).

\section{METHODS}

\section{Patients}

We included first-time enrolled RA and PsA patients from the prospective longitudinal observational multicentre NORwegian-Disease Modifying Anti-Rheumatic Drug (NORDMARD) study, ${ }^{9}$ starting synthetic and/or biologic DMARDs between 1 December 2000 and 6 November 2012 and followed until 1 May 2013 or until stopping DMARD medication. The RA and PsA diagnoses were given by the treating rheumatologist after clinical judgement. Analyses included baseline, 3- and 6-month follow-up. Written informed consent was obtained from each patient. The study was approved by the National Data Inspectorate and by the Regional Committee for Medical and Health Research Ethics in Eastern Norway. For comparison, we included SF-36 Norwegian normative data from 2323 individuals collected in 1996. $^{10}$

\section{Short Form-36 and short form-6 dimensions}

The Norwegian translation of SF-36 version 1, used in this study, is validated in Norwegian RA patients. ${ }^{11}$ PCS and MCS were calculated as described by Ware et al. ${ }^{12}$ Norm-based scale scores were computed by subtracting the general population's respective mean scale score from the 0-100 scale scores divided by the standard deviation of the data from the general population. Each of these values were multiplied by 10 and 50 was added, as described by Ware et al. ${ }^{13}$ SF-6D was calculated from SF-36 according to the algorithm developed by Brazier et al. ${ }^{14}$

\section{Statistics}

Demographic and baseline characteristics are shown as medians $\left(25^{\text {th }}, 75^{\text {th }}\right.$ percentiles $)$ for non-normally and means (SD) for normally distributed data. Continuous variables were compared using independent t-test, Mann-Whitney U-test or ANOVA, as appropriate. Prespecified age and gender-adjusted ANCOVA analyses were performed to compare PCS, MCS, scale scores and SF-6D between RA, PsA patients and general population controls at baseline, and between RA and PSA patients with and without additional adjustment for the respective 28-joint disease activity scores (DAS28) at baseline and after 3- and 6-month 
follow-up. Prespecified ANCOVA analyses were performed to compare changes in PCS, MCS, scale scores and SF-6D from baseline to 3 and 6 months follow-up, adjusted for age, gender and the respective baseline values. Radar diagrams were made to visualize differences in scale scores ( 0 worst, 100 best) between RA/PsA patients and the Norwegian general population. Statistical tests were performed using SPSS V.23.0 for Windows. The analyses were performed as completer analyses and without adjustment for multiple comparisons.

\section{RESULTS}

RA patients ( $n=3898)$, PsA patients $(n=1515)$ and general population controls $(n=2323)$ had mean (SD) age 55.9 (31.6)/ 48.1 (12.6)/ 44.9 (16.5) years and 71.4/ 50.3/ 51.3\% were females, respectively. Baseline mean (SD) DAS28 was worse in RA (4.9 (1.4)) vs. PsA (4.2 (1.3)) patients (online supplementary Table S1).

\section{Analyses adjusted for age and gender}

SF-36 PCS, MCS, scale scores and SF-6D were worse in RA and PsA patients compared with the general population (Table 1), but improved during follow-up (online supplementary Table S2).

Table 1 Unadjusted means (ANOVA) and estimated marginal means (ANCOVA) adjusted for age and gender of baseline SF-36 component summaries, norm-based scale scores and SF-6D utility scores

\begin{tabular}{|c|c|c|c|c|c|c|c|c|}
\hline \multicolumn{9}{|c|}{ Baseline } \\
\hline \multirow{2}{*}{$\begin{array}{c}\text { Component } \\
\text { summary/ scale score }\end{array}$} & \multicolumn{3}{|c|}{ Unadjusted analyses, mean (SD) } & \multirow{2}{*}{ p-value } & \multicolumn{3}{|c|}{$\begin{array}{c}\text { Adjusted analyses, } \\
\text { marginal means }(95 \% \mathrm{Cl})\end{array}$} & \multirow{2}{*}{$p$-value } \\
\hline & $\begin{array}{c}\text { General } \\
\text { population }\end{array}$ & RA & PsA & & $\begin{array}{c}\text { General } \\
\text { population }\end{array}$ & RA & PsA & \\
\hline PCS & $\begin{array}{c}50.0(10.1) \\
(n=2012)\end{array}$ & $\begin{array}{l}29.7(9.9) \\
(n=3898)\end{array}$ & $\begin{array}{l}30.5(9.5) \\
(n=1515)\end{array}$ & $\begin{array}{c}<0.001^{1,2} \\
0.02^{3}\end{array}$ & $\begin{array}{c}49.7 \\
(49.2-50.1)\end{array}$ & $\begin{array}{c}30.5 \\
(30.2-30.8)\end{array}$ & $\begin{array}{c}30.4 \\
(29.9-30.9)\end{array}$ & $\begin{array}{c}<0.001^{1,2} \\
0.69^{3}\end{array}$ \\
\hline MCS & $\begin{array}{c}50.0(10.2) \\
(n=2012)\end{array}$ & $\begin{array}{c}46.8(11.3) \\
(n=3898)\end{array}$ & $\begin{array}{c}47.1(11.4) \\
(n=1515)\end{array}$ & $\begin{array}{c}<0.001^{1,2} \\
0.59^{3}\end{array}$ & $\begin{array}{c}50.0 \\
(49.5-50.5)\end{array}$ & $\begin{array}{c}47.1 \\
(46.8-47.5)\end{array}$ & $\begin{array}{c}47.1 \\
(46.5-47.6)\end{array}$ & $\begin{array}{c}<0.001^{1,2} \\
0.90^{3}\end{array}$ \\
\hline SF-6D & $\begin{array}{c}0.80(0.14) \\
(n=2071)\end{array}$ & $\begin{array}{c}0.60(0.12) \\
(n=3787)\end{array}$ & $\begin{array}{c}0.61(0.12) \\
(n=1491)\end{array}$ & $\begin{array}{c}<0.001^{1,2} \\
0.17^{3}\end{array}$ & $\begin{array}{c}0.80 \\
(0.80-0.81)\end{array}$ & $\begin{array}{c}0.61 \\
(0.60-0.61)\end{array}$ & $\begin{array}{c}0.60 \\
(0.60-0.61)\end{array}$ & $\begin{array}{l}<0.001^{1,2} \\
0.45^{3}\end{array}$ \\
\hline Physical Functioning & $\begin{array}{c}49.4(10.8) \\
(n=2235)\end{array}$ & $\begin{array}{c}27.7(14.5) \\
(n=3898)\end{array}$ & $\begin{array}{c}30.5(13.5) \\
(n=1515)\end{array}$ & $<0.001^{1,2,3}$ & $\begin{array}{c}48.9 \\
(48.3-49.4)\end{array}$ & $\begin{array}{c}29.0 \\
(28.5-29.4)\end{array}$ & $\begin{array}{c}30.2 \\
(29.6-30.9)\end{array}$ & $\begin{array}{c}<0.001^{1,2} \\
0.001^{3}\end{array}$ \\
\hline Role Physical & $\begin{array}{c}49.6(10.3) \\
(n=2207)\end{array}$ & $\begin{array}{l}32.9(9.1) \\
(n=3898)\end{array}$ & $\begin{array}{l}34.0(9.9) \\
(n=1515)\end{array}$ & $\begin{array}{l}<0.001^{1,2} \\
0.001^{3}\end{array}$ & $\begin{array}{c}49.4 \\
(49.0-49.8)\end{array}$ & $\begin{array}{c}33.5 \\
(33.2-33.8)\end{array}$ & $\begin{array}{c}33.9 \\
(33.4-34.4)\end{array}$ & $\begin{array}{c}<0.001^{1,2} \\
0.16^{3}\end{array}$ \\
\hline Bodily Pain & $\begin{array}{c}49.6(10.3) \\
(n=2287)\end{array}$ & $\begin{array}{l}33.5(7.5) \\
(n=3898)\end{array}$ & $\begin{array}{l}33.6(7.2) \\
(n=1515)\end{array}$ & $\begin{array}{c}<0.001^{1,2} \\
0.96^{3}\end{array}$ & $\begin{array}{c}49.4 \\
(49.1-49.8)\end{array}$ & $\begin{array}{c}34.0 \\
(33.7-34.2)\end{array}$ & $\begin{array}{c}33.5 \\
(33.1-33.9)\end{array}$ & $\begin{array}{c}<0.001^{1,2} \\
0.07^{3}\end{array}$ \\
\hline General Health & $\begin{array}{c}49.6(10.3) \\
(n=2183)\end{array}$ & $\begin{array}{l}37.5(9.5) \\
(n=3898)\end{array}$ & $\begin{array}{l}36.8(9.7) \\
(n=1515)\end{array}$ & $\begin{array}{c}<0.001^{1,2} \\
0.02^{3}\end{array}$ & $\begin{array}{c}49.5 \\
(49.0-49.9)\end{array}$ & $\begin{array}{c}37.9 \\
(37.6-38.3)\end{array}$ & $\begin{array}{c}36.7 \\
(36.2-37.2)\end{array}$ & $<0.001^{1,2,3}$ \\
\hline Vitality & $\begin{array}{c}49.8(10.3) \\
(n=2270)\end{array}$ & $\begin{array}{c}39.6(10.1) \\
(n=3898)\end{array}$ & $\begin{array}{c}39.3(10.3) \\
(n=1515)\end{array}$ & $\begin{array}{c}<0.001^{1,2} \\
0.69^{3}\end{array}$ & $\begin{array}{c}49.9 \\
(49.5-50.3)\end{array}$ & $\begin{array}{c}40.4 \\
(40.1-40.8)\end{array}$ & $\begin{array}{c}39.4 \\
(38.9-39.9)\end{array}$ & $<0.001^{1,2,3}$ \\
\hline Social Functioning & $\begin{array}{c}49.5(10.5) \\
(n=2311)\end{array}$ & $\begin{array}{c}39.1(12.6) \\
(n=3898)\end{array}$ & $\begin{array}{c}39.5(12.3) \\
(n=1515)\end{array}$ & $\begin{array}{c}<0.001^{1,2} \\
0.59^{3}\end{array}$ & $\begin{array}{c}49.4 \\
(48.9-49.9)\end{array}$ & $\begin{array}{c}39.8 \\
(39.4-40.2)\end{array}$ & $\begin{array}{c}39.5 \\
(38.9-40.1)\end{array}$ & $\begin{array}{c}<0.001^{1,2} \\
0.40^{3}\end{array}$ \\
\hline
\end{tabular}




\begin{tabular}{|l|c|c|c|c|c|c|c|c|}
\hline Role Emotional & $\begin{array}{c}49.8(10.2) \\
(\mathrm{n}=2182)\end{array}$ & $\begin{array}{c}40.2(13.6) \\
(\mathrm{n}=3898)\end{array}$ & $\begin{array}{c}41.6(13.6) \\
(\mathrm{n}=1515)\end{array}$ & $\begin{array}{c}<0.001^{1,2} \\
0.002^{3}\end{array}$ & $\begin{array}{c}49.5 \\
(49.0-50.0)\end{array}$ & $\begin{array}{c}40.6 \\
(40.2-41.1)\end{array}$ & $\begin{array}{c}41.5 \\
(40.8-42.1)\end{array}$ & $\begin{array}{c}<0.001^{1,2} \\
0.03^{3}\end{array}$ \\
\hline Mental Health & $\begin{array}{c}49.8(10.3) \\
(\mathrm{n}=2255)\end{array}$ & $\begin{array}{c}44.3(11.5) \\
(\mathrm{n}=3898)\end{array}$ & $\begin{array}{c}44.7(11.1) \\
(\mathrm{n}=1515)\end{array}$ & $\begin{array}{c}<0.001^{1,2} \\
0.63^{3}\end{array}$ & $\begin{array}{c}49.8 \\
(49.4-50.3)\end{array}$ & $\begin{array}{c}44.8 \\
(44.5-45.2)\end{array}$ & $\begin{array}{c}44.7 \\
(44.2-45.3)\end{array}$ & $<0.001^{1,2}$ \\
$0.76^{3}$ \\
\hline
\end{tabular}

${ }^{1}$ General population vs. RA; ${ }^{2}$ General population vs. PsA; ${ }^{3}$ RA vs. PsA. MCS, mental component

Summary; PCS, physical component summary; PsA, psoriatic arthritis; RA, rheumatoid arthritis; SF-

6D, short form-6 dimensions utility scores

PCS and MCS were not statistically different in RA vs. PsA patients, while physical functioning was better and general health and vitality worse in PsA vs. RA patients at all time-points.

Bodily pain was similar between RA and PsA patients at baseline, but slightly worse in PsA patients at 3 and 6 months (table 1, online supplementary Table S2).

\section{Analyses adjusted for age, gender and DAS28}

In analyses adjusted for DAS28 in addition to age and gender, PsA patients had significantly worse PCS and SF-6D at all time-points compared to RA patients. Baseline MCS and all scale scores, except role emotional, were worse in PsA vs. RA. At 3 and 6 months bodily pain, general health, vitality, social functioning and mental health were worse and the remaining scale scores similar in PsA vs. RA (online supplementary Table S3).

\section{Longitudinal analyses adjusted for age, gender and baseline values of the respective scores} RA and PsA patients had similar improvements in PCS, MCS, all scale scores and SF-6D at 3 and 6 months, except for larger improvements in bodily pain, vitality and mental health in the RA patients (Table 2).

Table 2 Mean improvements from baseline until 3 and 6 months follow-up; unadjusted analyses (ANOVA) and analyses adjusted for age, gender and the respective baseline values (ANCOVA)

\begin{tabular}{|c|c|c|c|c|c|c|}
\hline \multirow{2}{*}{$\begin{array}{l}\text { Component } \\
\text { summary/ scale } \\
\text { score }\end{array}$} & \multicolumn{2}{|c|}{$\begin{array}{l}\text { Unadjusted analyses, } \\
\text { mean (SD) change from } \\
\text { baseline until } 3 \text { months }\end{array}$} & \multirow[t]{2}{*}{ p-value } & \multicolumn{2}{|c|}{$\begin{array}{c}\text { Adjusted analyses, } \\
\text { mean change }(95 \% \mathrm{Cl}) \text { from baseline } \\
\text { until } 3 \text { months }\end{array}$} & \multirow[t]{2}{*}{ p-value } \\
\hline & RA & PsA & & RA & PsA & \\
\hline PCS & $\begin{array}{c}4.5(9.2) \\
n=3095\end{array}$ & $\begin{array}{c}4.3(9.1) \\
n=1188\end{array}$ & 0.49 & $\begin{array}{c}4.7(4.3-5.0) \\
n=3081\end{array}$ & $\begin{array}{c}4.4(3.9-4.9) \\
\mathrm{n}=1183\end{array}$ & 0.42 \\
\hline MCS & $\begin{array}{c}1.9(10.6) \\
n=3095\end{array}$ & $\begin{array}{c}1.4(10.3) \\
\mathrm{n}=1188\end{array}$ & 0.13 & $\begin{array}{c}1.8(1.4-2.1) \\
n=3081\end{array}$ & $\begin{array}{c}1.5(1.0-2.0) \\
n=1183\end{array}$ & 0.41 \\
\hline SF-6D & $\begin{array}{c}0.04(0.1) \\
n=2321\end{array}$ & $\begin{array}{c}0.03(0.1) \\
n=876\end{array}$ & 0.12 & $\begin{array}{c}0.04(0.03-0.04) \\
n=2323\end{array}$ & $\begin{array}{c}0.03(0.03-0.04) \\
n=872\end{array}$ & 0.06 \\
\hline Physical Functioning & $\begin{array}{c}4.5(12.2) \\
\mathrm{n}=3211\end{array}$ & $\begin{array}{c}4.0(11.5) \\
n=1223\end{array}$ & 0.30 & $\begin{array}{c}4.6(4.2-5.0) \\
n=3197\end{array}$ & $\begin{array}{c}4.6(4.2-5.2) \\
n=1217\end{array}$ & 0.93 \\
\hline Role Physical & $\begin{array}{c}4.1(10.7) \\
\mathrm{n}=3192\end{array}$ & $\begin{array}{c}4.0(10.5) \\
n=1214\end{array}$ & 0.65 & $\begin{array}{c}4.2(3.8-4.6) \\
n=3178\end{array}$ & $\begin{array}{c}4.3(3.7-4.8) \\
n=1208\end{array}$ & 0.83 \\
\hline Bodily Pain & $\begin{array}{c}5.7(8.6) \\
n=3201\end{array}$ & $\begin{array}{l}5.1(8.0) \\
n=1220 \\
\end{array}$ & 0.02 & $\begin{array}{c}5.8(5.5-6.1) \\
n=3187\end{array}$ & $\begin{array}{c}5.1(4.6-5.5) \\
n=1214\end{array}$ & 0.004 \\
\hline
\end{tabular}




\begin{tabular}{|c|c|c|c|c|c|c|}
\hline General Health & $\begin{array}{l}1.3(8.1) \\
n=3163\end{array}$ & $\begin{array}{l}1.5(8.4) \\
n=1208\end{array}$ & 0.64 & $\begin{array}{c}1.4(1.1-1.7) \\
n=3149\end{array}$ & $\begin{array}{c}1.3(0.9-1.7) \\
n=1203\end{array}$ & 0.61 \\
\hline Vitality & $\begin{array}{c}3.6(10.2) \\
\mathrm{n}=3187\end{array}$ & $\begin{array}{l}2.9(9.9) \\
\mathrm{n}=1215\end{array}$ & 0.02 & $\begin{array}{c}3.8(3.5-4.2) \\
\mathrm{n}=3173\end{array}$ & $\begin{array}{c}2.8(2.3-3.4) \\
n=1209\end{array}$ & 0.002 \\
\hline Social Functioning & $\begin{array}{c}3.6(11.5) \\
\mathrm{n}=3215\end{array}$ & $\begin{array}{c}3.2(11.3) \\
\mathrm{n}=1222\end{array}$ & 0.23 & $\begin{array}{c}3.7(3.3-4.1) \\
n=3201\end{array}$ & $\begin{array}{c}3.3(2.8-3.9) \\
n=1216\end{array}$ & 0.31 \\
\hline Role Emotional & $\begin{array}{c}2.5(14.4) \\
\mathrm{n}=3175\end{array}$ & $\begin{array}{c}2.3(13.8) \\
n=1202\end{array}$ & 0.66 & $\begin{array}{c}2.2(1.8-2.7) \\
n=3161\end{array}$ & $\begin{array}{c}2.8(2.1-3.4) \\
n=1197\end{array}$ & 0.20 \\
\hline Mental Health & $\begin{array}{c}2.5(9.8) \\
n=3184\end{array}$ & $\begin{array}{l}1.7(9.1) \\
n=1215\end{array}$ & 0.02 & $\begin{array}{c}2.5(2.2-2.8) \\
n=3170\end{array}$ & $\begin{array}{c}1.9(1.4-2.4) \\
n=1210\end{array}$ & 0.008 \\
\hline \multirow{2}{*}{$\begin{array}{l}\text { Component } \\
\text { summary/ scale } \\
\text { score }\end{array}$} & \multicolumn{2}{|c|}{$\begin{array}{l}\text { Unadjusted analyses, } \\
\text { mean (SD) change from } \\
\text { baseline until } 6 \text { months }\end{array}$} & \multirow[t]{2}{*}{ p-value } & \multicolumn{2}{|c|}{$\begin{array}{c}\text { Adjusted analyses, } \\
\text { mean change }(95 \% \mathrm{Cl}) \text { from baseline } \\
\text { until } 6 \text { months }\end{array}$} & \multirow[t]{2}{*}{ p-value } \\
\hline & RA & PsA & & RA & PsA & \\
\hline PCS & $\begin{array}{l}5.3(9.6) \\
n=2616\end{array}$ & $\begin{array}{c}5.4(9.8) \\
n=1037\end{array}$ & 0.71 & $\begin{array}{c}5.6(5.2-6.0) \\
n=2605\end{array}$ & $\begin{array}{c}5.4(4.8-5.9) \\
n=1034\end{array}$ & 0.49 \\
\hline MCS & $\begin{array}{c}2.4(10.7) \\
n=2616\end{array}$ & $\begin{array}{c}2.0(10.6) \\
n=1037\end{array}$ & 0.35 & $\begin{array}{c}2.5(2.2-2.9) \\
\mathrm{n}=2605\end{array}$ & $\begin{array}{c}2.1(1.5-2.6) \\
n=1034\end{array}$ & 0.18 \\
\hline SF-6D & $\begin{array}{c}0.04(0.1) \\
n=1838\end{array}$ & $\begin{array}{c}0.04(0.1) \\
(n=723)\end{array}$ & 0.76 & $\begin{array}{c}0.04(0.04-0.05) \\
n=1832\end{array}$ & $\begin{array}{c}0.04(0.03-0.05) \\
n=721\end{array}$ & 0.42 \\
\hline Physical Functioning & $\begin{array}{c}5.5(12.6) \\
n=2689\end{array}$ & $\begin{array}{c}5.9(12.5) \\
n=1056\end{array}$ & 0.40 & $\begin{array}{c}5.8(5.4-6.3) \\
n=2676\end{array}$ & $\begin{array}{c}6.3(5.6-7.0) \\
n=1052\end{array}$ & 0.26 \\
\hline Role Physical & $\begin{array}{c}5.4(11.2) \\
n=2683\end{array}$ & $\begin{array}{c}5.4(11.6) \\
n=1053\end{array}$ & 0.93 & $\begin{array}{c}5.6(5.2-6.1) \\
n=2670\end{array}$ & $\begin{array}{c}5.6(5.0-6.2) \\
n=1048\end{array}$ & 0.91 \\
\hline Bodily Pain & $\begin{array}{l}6.3(8.9) \\
n=2688 \\
\end{array}$ & $\begin{array}{l}5.9(8.6) \\
n=1056 \\
\end{array}$ & 0.20 & $\begin{array}{c}6.7(6.3-7.0) \\
n=2675\end{array}$ & $\begin{array}{c}5.8(5.3-6.3) \\
n=1051\end{array}$ & 0.005 \\
\hline General Health & $\begin{array}{l}1.5(8.6) \\
n=2670\end{array}$ & $\begin{array}{l}1.6(8.4) \\
n=1053\end{array}$ & 0.81 & $\begin{array}{c}1.7(1.4-2.1) \\
n=2659\end{array}$ & $\begin{array}{c}1.3(0.9-1.8) \\
n=1050\end{array}$ & 0.17 \\
\hline Vitality & $\begin{array}{c}4.3(10.3) \\
n=2680\end{array}$ & $\begin{array}{c}4.1(10.5) \\
n=1055\end{array}$ & 0.62 & $\begin{array}{c}4.7(4.3-5.1) \\
n=2668\end{array}$ & $\begin{array}{c}3.9(3.3-4.4) \\
n=1051\end{array}$ & 0.02 \\
\hline Social Functioning & $\begin{array}{c}4.3(11.9) \\
n=2700\end{array}$ & $\begin{array}{c}4.4(11.2) \\
n=1059\end{array}$ & 0.81 & $\begin{array}{c}4.7(4.3-5.1) \\
n=2687\end{array}$ & $\begin{array}{c}4.4(3.8-5.0) \\
n=1054\end{array}$ & 0.39 \\
\hline Role Emotional & $\begin{array}{c}3.1(14.3) \\
\mathrm{n}=2670\end{array}$ & $\begin{array}{c}3.0(14.4) \\
\mathrm{n}=1049\end{array}$ & 0.90 & $\begin{array}{c}3.1(2.6-3.6) \\
n=2657\end{array}$ & $\begin{array}{c}3.4(2.7-4.2) \\
n=1045\end{array}$ & 0.44 \\
\hline Mental Health & $\begin{array}{c}3.0(10.0) \\
n=2677\end{array}$ & $\begin{array}{l}2.4(9.4) \\
n=1056\end{array}$ & 0.06 & $\begin{array}{c}3.2(2.9-3.6) \\
n=2665\end{array}$ & $\begin{array}{c}2.5(2.0-3.0) \\
n=1052\end{array}$ & 0.02 \\
\hline
\end{tabular}

$\mathrm{Cl}$, confidence interval; SD, standard deviation; MCS, mental component summary; PCS, physical Formatted: Norwegian (Bokmål) component summary; PsA, psoriatic arthritis; RA, rheumatoid arthritis; SF-6D, short form- 6 dimensions utility score

\section{Graphical comparisons of scale scores}

Estimated marginal means of baseline, 3- and 6-month SF-36 scale scores adjusted for age and gender (Figure) as well as DAS28 (figure not shown) were impaired in RA and PsA 
patients compared with the general population, but showed only small differences between RA and PsA patients.

\section{DISCUSSION}

In this large prospective observational multicentre study SF-36 PCS, MCS as well as the utility measure SF-6D were comparable between RA and PsA patients at baseline and at 6 months follow-up in age and gender-adjusted analyses. Furthermore, PsA patients had worse general health and vitality, but better physical functioning at all time-points, as well as more bodily pain at 3 and 6 months. With adjustment for DAS28 in addition to age and gender, PsA vs. RA patients had worse PCS, SF-6D, general health, vitality, bodily pain, social functioning and mental health at all time-points.

Further, the study shows that levels of physical HRQoL were more impaired than mental HRQoL in patients with RA and PsA compared with Norwegian general population controls. Improvements in scale scores from baseline until 3 and 6 months were similar between RA and PsA patients during treatment with DMARDs, except for larger improvements in bodily pain, vitality and mental health in RA.

We have chosen to present norm-based scale scores to facilitate interpretation and comparison across different countries and populations. ${ }^{13,15}$ Still, interpretation of the findings is not straight-forward; the statistically significant differences between RA and PsA patients may not always be clinically significant. As visualized in radar diagrams (Figure) the differences in scale scores between RA and PsA patients are small. Still, for e.g. general health the difference between RA and PsA patients at different time-points is of similar magnitude to the improvement in general health from baseline until 6 months both for RA and PsA patients and hence of probable clinical significance.

The study underlines the severe impact of both RA and PSA on HRQoL reflected through SF36 , which is in line with a smaller observational study ${ }^{3}$ as well as clinical trials. ${ }^{4,16,17}$ Furthermore, the study highlights the relatively stronger impairment of HRQoL in PsA compared with RA patients, when also taking into consideration levels of joint inflammation, as measured by DAS28. Notably, also according to PsA patients' perspective HRQoL is of great importance. ${ }^{1}$ The severe impact of PsA on HRQoL may possibly be explained by extraarticular inflammatory affection of e.g. skin or entheses. However, NOR-DMARD does not have data on these disease manifestations. We were therefore unable to identify which disease manifestations that contributed most to the reduced HRQoL in PsA. Of note, general health and vitality were worse in PsA compared with RA patients in all adjusted analyses at all time-points. RA as well as PsA patients reported better mental than physical HRQoL, which is in line with smaller observational studies on SF-36, ${ }^{3}$ as well as with clinical trials. ${ }^{4,16}$ Interestingly, the RA and PsA scale score profiles (Figure) were similar to the RA scale score profile found by Strand et al. ${ }^{16}$

Furthermore, the study is partly in line with two considerably smaller NOR-DMARD studies reporting better 6-month improvements in bodily pain and vitality, but not mental health in 
RA vs. PsA patients. ${ }^{18,19}$ These studies did not include general population controls. The generic SF-36 facilitated comparison of HRQoL across diseases, although it might capture somewhat different aspects of HRQoL than disease-specific measures like e.g. the psoriatic arthritis quality of life (PsAQoL). ${ }^{20}$

Limitations of the study include lack of $66 / 68$ joint count as well as lack of measures of PsA inflammatory activity other than arthritis (e.g. skin involvement, enthesitis, dactylitis and spondylitis), which may have led to underestimation of disease activity in PsA as used in the DAS28-adjusted analyses. Lack of adjustment for comorbidities may have biased the results, although partly corrected for by the age-adjustment. Furthermore, completer analyses may have affected the generalisability of the results.

The major strength of the study is the prospective observational multicentre design including large cohorts of RA and PsA patients over a long time span, as well as Norwegian general population controls. To our knowledge this is the largest prospective observational study comparing SF-36 between RA and PsA patients, and the first to compare SF-36 component summaries, scale scores as well as SF-6D between RA and PsA patients and general population controls in the same analyses.

In conclusion, HRQoL was overall similar in patients with RA and PsA and worse compared with general population controls. Most importantly, we found a tendency to worse scores in PsA than in RA which may indicate that unmet needs in treatment may be larger in PsA.

Acknowledgements: The authors thank the patients for participating in this study and the local rheumatology staff for data collection.

Competing interests: All authors have declared that no competing interests exist.

Contributors: BM, TU, JS, DVDH, HBH, EKK and TKK were responsible for study design. TU, AW, GB, ER, FK and TKK were responsible for data acquisition. BM analysed the data and wrote the manuscript. All authors critically revised the manuscript and approved the final version.

Funding: The study was funded through a clinical research fellowship from Diakonhjemmet Hospital, originating from South-Eastern Health Authority and through a grant from Grethe Harbitz' Legacy. Datacollection in NOR-DMARD was partly funded through unrestricted grants from Abbvie, BMS, MSD, Pfizer (Wyeth), Roche and UCB.

\section{REFERENCES}

1. Orbai AM, de Wit M, Mease PJ, et al. Updating the Psoriatic Arthritis (PsA) Core Domain Set: A Report from the PsA Workshop at OMERACT 2016. J Rheumatol 2017. 
2. van Tuyl LH, Sadlonova M, Hewlett S, et al. The patient perspective on absence of disease activity in rheumatoid arthritis: a survey to identify key domains of patient-perceived remission. Ann Rheum Dis 2017;76:855-61.

3. Salaffi F, Carotti M, Gasparini S, et al. The health-related quality of life in rheumatoid arthritis, ankylosing spondylitis, and psoriatic arthritis: a comparison with a selected sample of healthy people. Health Qual Life Outcomes 2009;7:25.

4. Strand V, Sharp V, Koenig AS, et al. Comparison of health-related quality of life in rheumatoid arthritis, psoriatic arthritis and psoriasis and effects of etanercept treatment. Ann Rheum Dis 2012;71:1143-50.

5. Husted JA, Gladman DD, Farewell VT, et al. Health-related quality of life of patients with psoriatic arthritis: a comparison with patients with rheumatoid arthritis. Arthritis Rheum 2001;45:1518.

6. Uhlig T, Loge JH, Kristiansen IS, et al. Quantification of reduced health-related quality of life in patients with rheumatoid arthritis compared to the general population. J Rheumatol 2007;34:1241-7.

7. Sokoll KB, Helliwell PS. Comparison of disability and quality of life in rheumatoid and psoriatic arthritis. J Rheumatol 2001;28:1842-6.

8. Borman $P$, Toy GG, Babaoglu S, et al. A comparative evaluation of quality of life and life satisfaction in patients with psoriatic and rheumatoid arthritis. Clin Rheumatol 2007;26:330-4.

9. Kvien TK, Heiberg, Lie E, et al. A Norwegian DMARD register: prescriptions of DMARDs and biological agents to patients with inflammatory rheumatic diseases. Clin Exp Rheumatol 2005;23:S188-94.

10. Loge JH, Kaasa S. Short form 36 (SF-36) health survey: normative data from the general Norwegian population. Scand J Soc Med 1998;26:250-8.

11. Kvien TK, Kaasa S, Smedstad LM. Performance of the Norwegian SF-36 Health Survey in patients with rheumatoid arthritis. II. A comparison of the SF-36 with disease-specific measures. J Clin Epidemiol 1998;51:1077-86.

12. Ware JE, Jr., Kosinski M, Bayliss MS, et al. Comparison of methods for the scoring and statistical analysis of SF-36 health profile and summary measures: summary of results from the Medical Outcomes Study. Med Care 1995;33:As264-79.

13. Ware JE, Jr., Kosinski M. SF-36 physical and mental health summary scales : a manual for users of version 1 QualityMetric Inc., 2001.

14. Brazier J, Roberts J, Deverill M. The estimation of a preference-based measure of health from the SF-36. J Health Econ 2002;21:271-92.

15. Ware JE, Jr. SF-36 health survey update. Spine (Phila Pa 1976) 2000;25:3130-9.

16. Strand V, Singh JA. Newer biological agents in rheumatoid arthritis: impact on health-related quality of life and productivity. Drugs 2010;70:121-45.

17. Frendl DM, Ware JE, Jr. Patient-reported functional health and well-being outcomes with drug therapy: a systematic review of randomized trials using the SF-36 health survey. Med Care 2014;52:439-45.

18. Lie $E$, van der Heijde D, Uhlig $T$, et al. Effectiveness and retention rates of methotrexate in psoriatic arthritis in comparison with methotrexate-treated patients with rheumatoid arthritis. Ann Rheum Dis 2010;69:671-6.

19. Heiberg MS, Koldingsnes W, Mikkelsen K, et al. The comparative one-year performance of antitumor necrosis factor alpha drugs in patients with rheumatoid arthritis, psoriatic arthritis, and ankylosing spondylitis: results from a longitudinal, observational, multicenter study. Arthritis Rheum 2008;59:234-40.

20. McKenna SP, Doward LC, Whalley D, et al. Development of the PsAQoL: a quality of life instrument specific to psoriatic arthritis. Ann Rheum Dis 2004;63:162-9. 